\title{
Quadriparesis as an unusual manifestation of chronic subdural haematoma
}

\author{
F LESOIN, A DESTEE, M JOMIN, P WAROT, SG WILSON \\ From the Departments of Neurology and Neurosurgery, Centre Hospitalier Universitaire de Lille, France and \\ Atkinson Morley's Hospital, Wimbledon, UK
}

SUMMARY Chronic subdural haematomas are frequently seen in neurosurgical practice, but because of the multitude of their clinical presentations, appropriate diagnosis is often not made in the early stages. The cases reported concern a 62-year-old male and a 54-year-old woman, both presenting in an unusual manner, namely a progressive quadriparesis.

In a variety of clinical situations, blood may accumulate between the brain and its surrounding dural covering to produce a haematoma. The clinical manifestations which occur as a consequence of the dynamic alteration in intracranial pressure/volume relationships in response to the changing pathology of the haematoma are quite varied and the diagnosis is easily missed. This is particularly so in the case of chronic subdural haematomas as the initial injury may be minimal or indeed forgotten. It may present with episodic drowsiness, confusion and a mild hemiparesis although specific neurological deficits like dysphasia and homonymous hemianopia are rare. We describe two cases illustrating a most unusual presentation of this elusive and clinically baffling condition.

\section{Case reports}

Case 1

A 62-year-old man fell on 12 December 1981 suffering a minor direct impact of the right parieto-occipital region, without loss of consciousness. The next day, he complained of a non specific generalised headache but was otherwise well. Over the next few weeks his family and friends observed a pronounced change in the patients behaviour, in that he became progressively apathetic and withdrawn. The headache persisted and he consulted his physician, who reported no specific signs. His fundi were normal and both his skull radiographs and EEG were normal. Three weeks after his head injury he developed vague difficulties

Address for reprint requests: F Lesoin, MD, Service de Neurochirurgie Centre Hospitalier Universitaire de Lille, 59000 France.

Received 30 July 1982 and in revised form 24 March 1983. Accepted 27 March 1983 in walking with a mild global weakness of both legs and on 9 March he experienced rapid difficulty in moving all four extremities. Over the next three days he deteriorated, developed a quadriplegia, and was admitted to hospital. Apart from previous hypertension and a history of alcohol abuse, there were no other relevant factors and no other recent head injury. On admission he was only semiconscious and had a quadriplegia with weakness most marked in the right leg and the left arm. Sensory testing was not possible because of his poor conscious level. An emergency CT scan showed bilateral chronic subdural haematomas with some increased density posteriorly suggestive of recent haemorrhage (fig 1). Bilateral burr holes were performed and the dura opened to reveal extensive chronic subdural collections of blood. Some fresh blood was present indicative of recent bleeding. The chronic subdural haematoma was evacuated and bilateral drains were clipped in place. Within 24 hours the patient improved dramatically. He became fully alert and orientated with some improvement in the quadriparesis. Radiographs, on which the positions of the clips were visible, showed that cerebral re-expansion had occurred. He was given intravenous fluids and hydrocortisone $(250 \mathrm{mg} /$ day $)$ for four days and recovered completely over the succeeding two weeks.

\section{Case 2}

A 55-year-old woman with no significant past medical history, was apparently completely well until 8 January 1982 when she bent forwards to get out of the passenger seat of a car and suddenly developed weakness of the right leg. This was global and was not associated with any sensory disturbance and completely settled over eight to ten minutes. She woke up that night to go to the lavatory, fell down and found that she could not use her right arm or right leg and was admitted to hospital. She was fully alert and orientated in space and time with no impairment of higher intellectual function. Her cranial nerves and fundi were normal. She had no movement whatsoever in her right lower limb and movement of the right upper limb was 


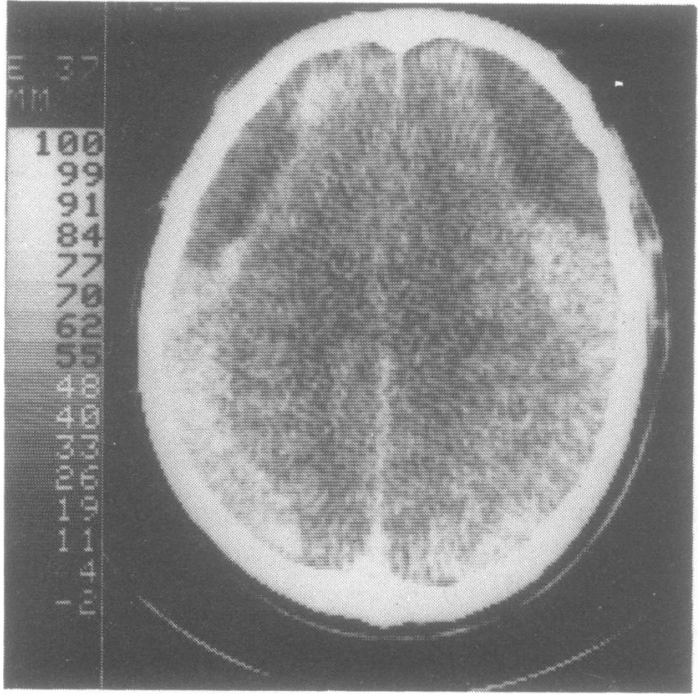

Fig 1 Enhanced CT scan demonstrating bilateral chronic subdural haematomas with hyperdensity of the posterior part indicative of recent haemorrhage. limited to grade 2 power of finger flexion. Reflexes were pathologically brisk in right upper and lower limbs with an extensor plantar on the right. Routine biochemical investigations, skull and cervical spine radiographs were normal. She awoke on 13 January to find that she now had a complete flaccid quadriplegia and was transferred for neurological assessment. Tone was spastic in all four limbs with brisk reflexes and bilateral extensor plantars. Higher intellectual functions were completely normal and she had no cranial nerve abnormalities. EEG showed minor bilateral slow wave activity and a CT scan was originally reported as normal. Myelography was performed to rule out a high cervical cord lesion and was completely normal. By 20 January she had become mildly confused and fundal examination suggested early bilateral papilloedema and there was now a prominent pout reflex and brisk jaw jerk. She still had a complete spastic quadriplegia. The CT scans were reviewed and the suggestion of isodense bilateral subdurals was made and she had bilateral carotid arteriography which confirmed extensive bilateral chronic subdural haematoma (fig 2a, b). Her relatives later mentioned a minor head injury on 20 December 1981 when she slipped and fell backwards and struck her head. There was no apparent injury at that time and the patient had forgotten the incident. Bilateral posterior and frontal burr holes were made under local anaesthetic with evacuation of $70 \mathrm{ml}$ of fluid on both right and left sides. Fine finger movements returned in both hands within a matter of minutes. The pattern of recovery was most unusual in that power
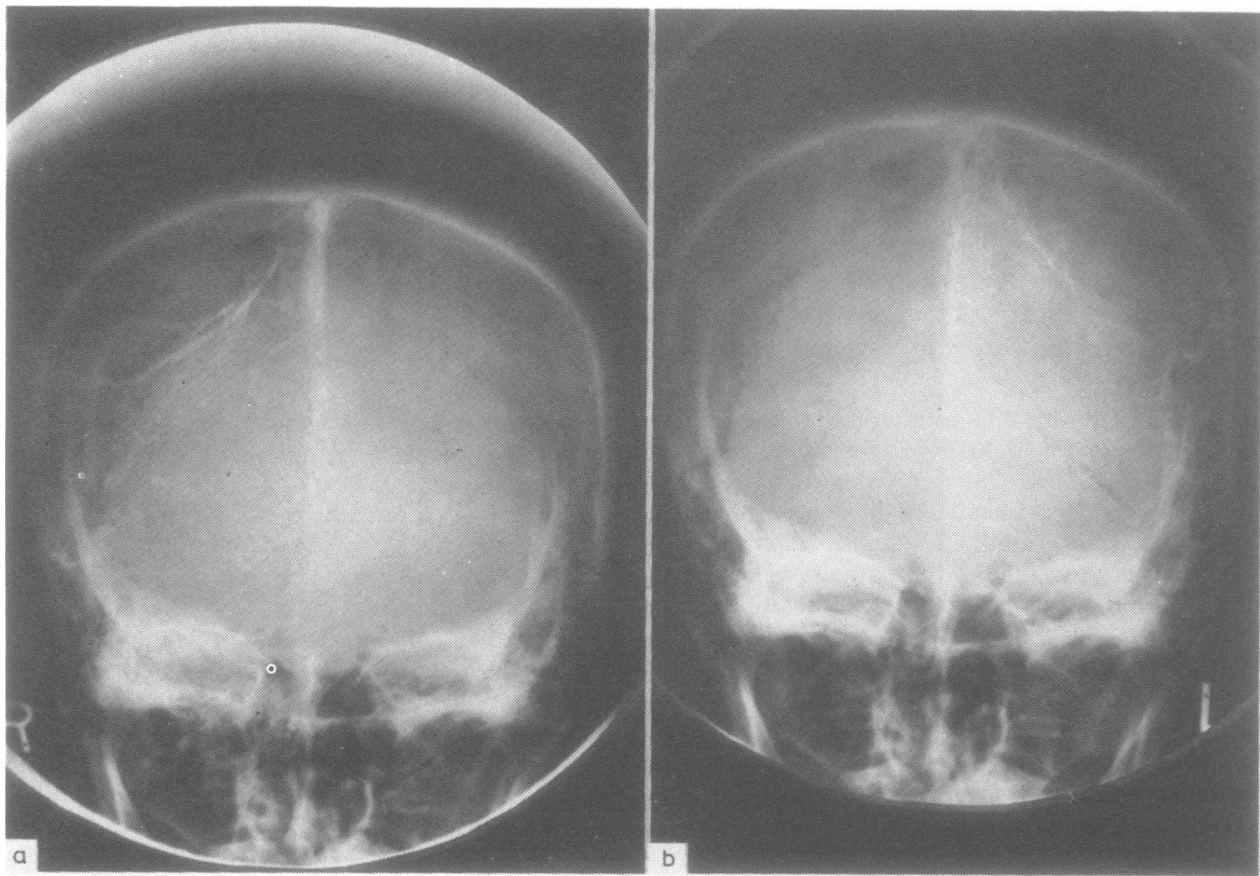

Fig 2 (a) Right and (b) left carotid arteriogram demonstrating an extensive subdural haematoma. 
returned distally at first and later the improvement became more proximal. By 27 January she had no movement of the shoulders but grade 4 in both elbow flexion and extension and grade 4 wrist extension. By 5 February all her signs had completely disappeared.

\section{Discussion}

Of all the complications of cerebral trauma chronic subdural haematoma is perhaps the most misleading. Indeed it can apparently develop in the absence of any known trauma. Its clinical features may occur long after the incident (often many weeks after, as in the above reported cases). The initial injury is often so slight that it is not considered important, or even forgotten by the patient.

Among the "classical" manifestations of chronic subdural haematoma are: progressive neurological deficits associated with intracranial hypertension, recent appearance of psychiatric troubles with or without neurological deficit, recent appearance of epilepsy and isolated intracranial hypertension. The list of less common, but far from rare, manifestations is much longer. Laplane and Philippon ${ }^{1}$ reported one case in which the only manifestation was a unilateral external occular motor paralysis. De Preux $^{2}$ observed a patient who had developed a paraparesis in the absence of any cerebral symptoms. Similarly, Shields ${ }^{3}$ presented a patient with an isodense subdural haematoma who presented with a paraparesis. Patients presenting with quadriparesis are rare, indeed, such a mode of presentation was not observed in 120 cases published by Debois ${ }^{4}$ and 114 cases presented by Cameron. ${ }^{5}$

The deficit in the arms can perhaps be explained by direct compression or distortion of the cerebral hemispheres. However this does not account for severe motor weakness in the legs as the cortical areas responsible for the lower limbs would be relatively protected from the direct effect of compression. The paraparesis may have developed from ischaemia of the appropriate cortical areas supplied by the anterior cerebral arteries. This would necessitate marked frontal lobe ischaemia, which should be accompanied by an alteration in conscious level. It has been proposed that the quadriplegia might be a manifestation of brain stem compression or distortion although it seems to develop in the absence of any specific or significant brain stem symptoms or signs. Finally it has been postulated that the quadriplegia might result from a diminished venous drainage from the Rolandic zone resulting from compression of the veins draining from this region to the superior longitudinal sinus. ${ }^{3}$
Although many of the symptoms of chonic subdural haematoma progress slowly, the course may be stepwise, as in these cases, with apparent abrupt periods of sudden deterioration. The reasons for this are uncertain but may result from acute re-bleeding into the capsule of the subdural haematoma (Apfel-Baum). ${ }^{6}$ Since the advent of the CT scan, there have been many observations of hypodense zones suggestive of bleeding in the past, and hyperdense zones indicative of recent bleeding. Sato and Suzuki ${ }^{7}$ have shown that the capsule of the chronic subdural haematoma has a dense capillary vasculature which increases as the chronic subdural haematoma ages. They postulate that the increase in size of the chronic subdural haematoma may result from repetitive re-bleeding into the capsule from the ever growing capillary bed. This is an interesting suggestion and may help to explain the apparent sudden phases of clinical deterioration which occurred in these two cases and in many of the reported cases.

Chronic subdural haematomas can present in a number of unusual ways. They may develop in the absence of any history of head injury, and without any obvious change in higher functions. They can present with paraplegia and as in these cases with quadriplegia. The diagnosis of chronic subdural haematoma must be considered when investigating cases of paraplegia and quadriplegia of uncertain etiology.

\section{References}

'Laplane D, Philippon J. L'hématome sous dural chronique de l'adulte. Rev Prat 1971;21:1225-37.

${ }^{2}$ De Preux JMD, Stephanov SMD. Paraparesis due to bilateral subdural haematoma. Surg Neurol 1981;16:346.

${ }^{3}$ Shields CB, Stites B, Garretson HD. Isodense subdural haematoma presenting with paraparesis. J Neurosurg 1980;52:712-4.

${ }^{4}$ Debois V, Combelles G, Dhellemmes P, Bousquet C, Jomin $M$. L'hématome sous dural chronique de L'adulte (à propos de 120 observations). Lille Médical 1978;23:522-8.

${ }^{5}$ Cameron MM. Chronic subdural haematoma: A review of 114 cases. J Neurol Neurosurg Psychiatry 1978;41:834-9.

- Apfelbaum RI, Guthrelch AN, Schulman R. Experimental production of subdural haematomas. $J$ Neurosurg 1974;40:336-46.

${ }^{7}$ Sato S, Suzuki S. Ultrastructural observations of the capsule of chronic subdural haematoma in various clinical stages. J Neurosurg 1975;43:569-78. 